\title{
Surgical treatment of blood blister-like aneurysms of the supraclinoid internal carotid artery with extracranial-intracranial bypass and trapping
}

\author{
Mustafa K. Başkaya, M.D., Azam S. Ahmed, M.D., ÖZkan Ateş, M.D., \\ AND DAVID NiEMANN, M.D. \\ Department of Neurological Surgery, University of Wisconsin, Madison, Wisconsin
}

\begin{abstract}
Object. Blood blister-like aneurysms (BBAs) arise from the supraclinoid internal carotid artery (ICA) at nonbranching sites. These aneurysms are challenging to treat primarily with either surgical clip placement or endovascular therapy. The authors describe a series of 4 patients who presented with high-grade subarachnoid hemorrhage $(\mathrm{SAH})$ due to a BBA, which was treated with an extracranial-intracranial (EC-IC) bypass followed by trapping of the aneurysm.

Methods. Four patients presented with SAH due to a BBA of the ICA. Three of these patients were treated with an endovascular procedure; following the vasospasm period, definitive treatment with EC-IC bypass followed by trapping of the aneurysmal parent vessel was performed.

Results. Two of the patients who were treated endovascularly suffered rebleeding prior to bypass and trapping. Three of the 4 patients had a good outcome (modified Rankin Scale Score 1 or 2), and 1 patient who suffered 2 episodes of rebleeding died.

Conclusions. Treatment of BBAs of the ICA remains difficult, particularly in the setting of high-grade SAH. Patients with this challenging condition often require multiple procedures and have a high incidence of rebleeding. Definitive treatment of these aneurysms consists of EC-IC bypass and surgical or endovascular trapping.

(DOI: 10.3171/FOC/2008/24/2/E13)
\end{abstract}

\section{KEY WoRDS • blood blister-like aneurysm • bypass procedure • supraclinoid internal carotid artery - trapping}

$\mathrm{S}$ UPRACLINOID ICA aneurysms that do not occur at branch points may be saccular or blood blister-like. The latter are notoriously difficult to treat by any means because of their small size, fragile walls, lack of an aneurysm neck, and tendency to avulse with minimal manipulation. . $3,3,8,10,14-16$ Surgical methods of treating BBAs of the supraclinoid ICA include primary clip occlusion with vessel reconstruction, surgical wrapping, Sundt clip placement, and ICA trapping with or without EC-IC bypass. ${ }^{10,15,20}$ Recently, the endovascular treatment results for BBAs in the ICA have been reported. Combined surgical and endovascular therapies have been reported as well. ${ }^{11}$ Endovascular methods used to treat BBA include primary coil embolization, with or without adjunctive devices such as stents and balloons, or endovascular trapping after

Abbreviations used in this paper: $\mathrm{AChA}=$ anterior choroidal artery; $\mathrm{BBA}=$ blood blister-like aneurysm; BTO = balloon test occlusion; $\mathrm{CT}$ = computed tomography; $\mathrm{DS}=$ digital subtraction; EC-IC = extracranial-intracranial; ECA = external carotid artery; $\mathrm{ICA}=$ internal carotid artery; $\mathrm{MCA}=$ middle cerebral artery; $\mathrm{mRS}=$ modified Rankin Scale; PCA = posterior cerebral artery; PCoA = posterior communicating artery; $\mathrm{SAH}=$ subarachnoid hemorrhage.
$\mathrm{BTO} .^{2}$ The periprocedural morbidity and mortality rates are high with either modality of treatment, and aneurysm growth and rebleeding are both common if the aneurysm is treated primarily. $3,4,14,16,20$ Although the optimal method of treatment must be individualized to the patient, the high failure rate after primary treatment alone makes definitive treatment with surgical or endovascular trapping, with or without EC-IC bypass, an attractive option.

\section{Clinical Materials and Methods}

We reviewed the electronic and paper records, imaging studies, and operative and endovascular reports of the 4 patients in this study. Patient data are summarized in Table 1. The patients presented with SAH between January 2006 and May 2007 due to a BBA of the ICA. The diagnosis was confirmed using DS angiography. Endovascular procedures were performed in 3 patients prior to bypass and trapping. These procedures consisted of stent placement, coil placement with balloon remodeling, and initial coil treatment of the aneurysm. In 2 patients collateral circulation was assessed using BTO prior to definitive treatment, but clinical assessment was not definitive because of their poor clinical condition. Trapping was performed after bypass in 
TABLE 1

Characteristics of 4 patients undergoing surgery for BBAs*

\begin{tabular}{|c|c|c|c|c|c|c|c|}
\hline $\begin{array}{l}\text { Case } \\
\text { No. }\end{array}$ & $\begin{array}{l}\text { Age (yrs), } \\
\text { Sex }\end{array}$ & $\begin{array}{c}\text { H \& H/ } \\
\text { Fisher Grades }\end{array}$ & $\begin{array}{l}\text { BBA Size } \\
(\mathrm{mm})\end{array}$ & Initial Treatment & Rebled & $\begin{array}{c}\text { Method of } \\
\text { Vessel Closure }\end{array}$ & $\begin{array}{c}\text { mRS Score/ } \\
\text { FU (mos) }\end{array}$ \\
\hline 1 & 23, M & $\mathrm{IV} / 3$ & 3.5 & balloon-remodeled coil & yes & clip & $1 / 3$ \\
\hline 2 & $57, \mathrm{M}$ & $\mathrm{IV} / 3$ & 3 & dual stent \& coil & yes (twice) & coil & $6 / 0$ \\
\hline 3 & $24, \mathrm{M}$ & $\mathrm{IV} / 3$ & 2 & none & no & coil & $1 / 3$ \\
\hline 4 & $56, \mathrm{~F}$ & $\mathrm{IV} / 3$ & 1.8 & dual stent & no & clip & $1 / 1$ \\
\hline
\end{tabular}

$* \mathrm{FU}=$ follow-up; $\mathrm{H} \& \mathrm{H}=$ Hunt and Hess.

all cases, and 2 patients underwent endovascular trapping by parent vessel occlusion. In the other 2 patients, trapping was accomplished with clips. All patients were treated aggressively for vasospasm with medical therapy, intraarterial verapamil infusion, and angioplasty when necessary. Clinical outcomes were assessed using the mRS. ${ }^{13}$

\section{The EC-IC Bypass Procedure}

In preparation for bypass, all patients were given $325 \mathrm{mg}$ enteric-coated aspirin on the day of the procedure. All patients received $10 \mathrm{mg}$ dexamethasone intravenously and 1 $\mathrm{g} / \mathrm{kg}$ mannitol intravenously immediately before incision. After pterional craniotomy, the MCA and its major branches were isolated. The cervical ECA was exposed via an incision along the medial side of the sternocleidomastoid muscle. A pediatric chest tube was used to create a preauricular subcutaneous tunnel between the 2 incisions through which the harvested radial artery was passed. The radial artery was used as the bypass conduit in all cases and had been prepared with papaverine. The orientation of the graft was carefully monitored such that the proximal graft was anastomosed with the ECA and the distal end of the graft was anastamosed with the intracranial vessel. A thin white piece of rubber was placed under the recipient dominant $\mathrm{M}_{2}$ segment for anastomosis. After intravenous administration of $2000 \mathrm{U}$ systemic heparin, an $\sim 1.5-\mathrm{cm}$ length of the $\mathrm{M}_{2}$ segment was trapped with a temporary clip. All EC-IC bypasses were performed between the ECA and the larger of the $2 \mathrm{M}_{2}$ branches of the MCA. An arteriotomy $\sim 5 \mathrm{~mm}$ in length was created. The radial artery graft was anastomosed first to the $\mathrm{M}_{2}$ segment with 10-0 nylon sutures. Temporary clips on the $\mathrm{M}_{2}$ segment were released. The cervical ECA was occluded proximally and distally with temporary clips. After the arteriotomy on the cervical ECA, the radial artery graft was anastomosed to the ECA with 8-0 nylon sutures. After the completion of the anastomosis, the temporary clips were removed. Flow through the graft was assessed using Doppler ultrasonography and a flow probe. All patients commenced a regimen of aspirin therapy indefinitely.

Trapping of the aneurysm was accomplished either by placing a clip in the ICA immediately proximal and distal to the aneurysm, or by coil embolization of the aneurysm segment.

\section{Endovascular ICA Trapping}

Systemic heparinization was used to achieve an activated clotting time between 2 and 2.5 times that of the patient's baseline. An OB-5 balloon catheter (Boston Scientific, Inc.) was placed into the ICA. The balloon was inflated to avoid distal coil migration and to allow continuous heparinized saline infusion. A microcatheter and
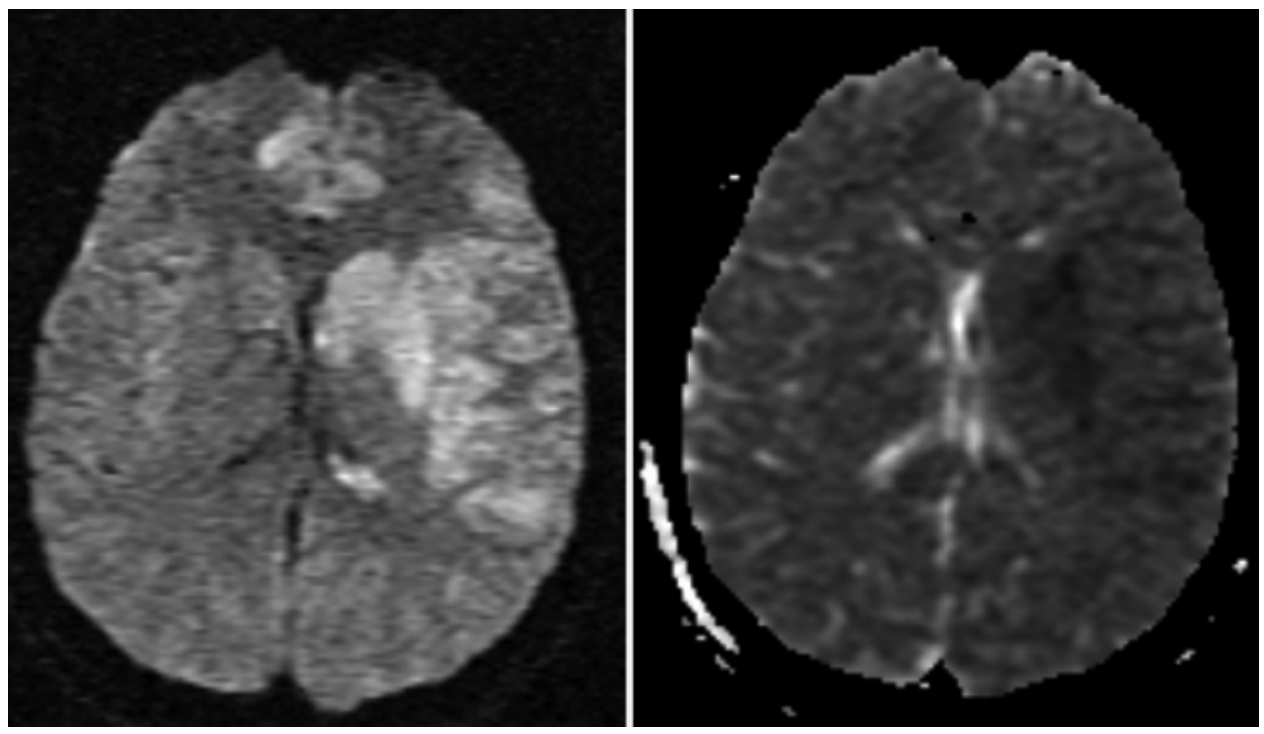

FIG. 1. Case 1. Diffusion weighted (left) and apparent diffusion coefficient ( right) magnetic resonance images of the brain demonstrating left perisylvian and striatal infarction with surrounding penumbra.. 


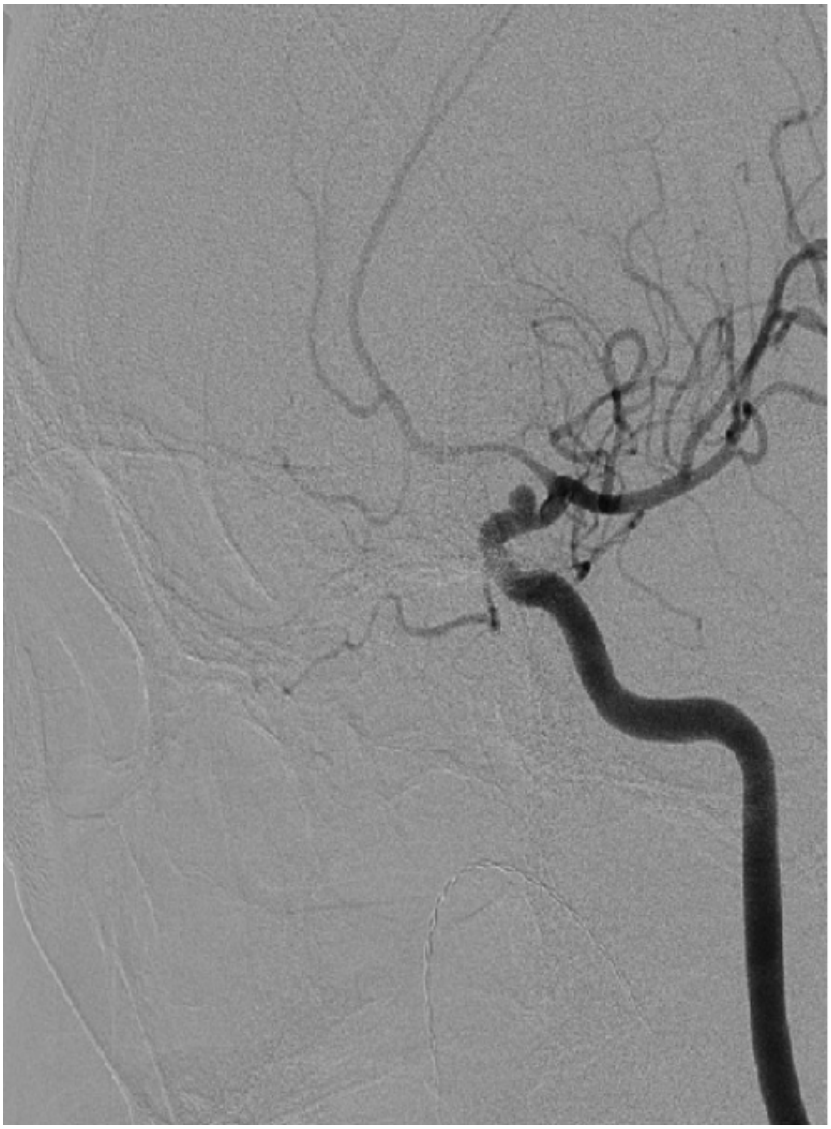

FIG. 2. Case 1. Digital subtraction angioram demonstrating a 3.5-mm BBA of the anteromedial wall of the left supraclinoid ICA.

microwire were inserted through the balloon catheter, to attain a position distal to the aneurysm. A diagnostic catheter was placed in the contralateral ICA so that control angiography could be performed to adequately assess the collateral circulation and the carotid artery terminus. Coils were then placed in the parent vessel distally to proximally across the aneurysm segment so that as dense as possible a coil mass was created.

\section{Summary of Cases}

\section{Case 1}

Presentation and Examination. This 23-year-old man was in a comatose state and had a Hunt and Hess Grade IV and a Fisher Grade 3 SAH. He had localized pain in his left upper extremity and was hemiplegic on his right side. Brain $\mathrm{CT}$ and magnetic resonance images showed diffuse $\mathrm{SAH}$ and left perisylvian and striatal infarction with surrounding penumbra (Fig. 1). Cerebral angiography confirmed the diagnosis of a $3.5-\mathrm{mm}$ BBA of the anteromedial wall of the left supraclinoid ICA (Fig. 2).

First Operation. Because of the patient's high-grade SAH and left-sided infarction we elected to perform endovascular coil placement with balloon remodeling of the aneurysm to allow the patient to recover from the $\mathrm{SAH}$ as well as to treat the vasospasm (Fig. 3). During this procedure, coils were pushed through the extent of the dome of the aneurysm; however, CT scanning did not reveal worsened SAH. The patient was treated for symptomatic vasospasm endovascularly with intraarterial verapamil on 2 occasions.

Second Operation. On Day 16, the patient's condition had improved to the point that he opened his eyes to verbal stimuli, but he remained densely hemiparetic and aphasic. Repeated angiography showed expansion of the aneurysm (Fig. 4). On posthemorrhage Day 17, the patient underwent an ECA-M $\mathrm{M}_{2}$ high-flow bypass and surgical trapping of the aneurysm. Three clips were placed to trap the aneurysm as follows: the first was placed on the ICA, distal to the ophthalmic artery origin; the second was placed immediately proximal to the AChA origin; and the third was placed at the origin of the PCoA. Although the clip on the AChA

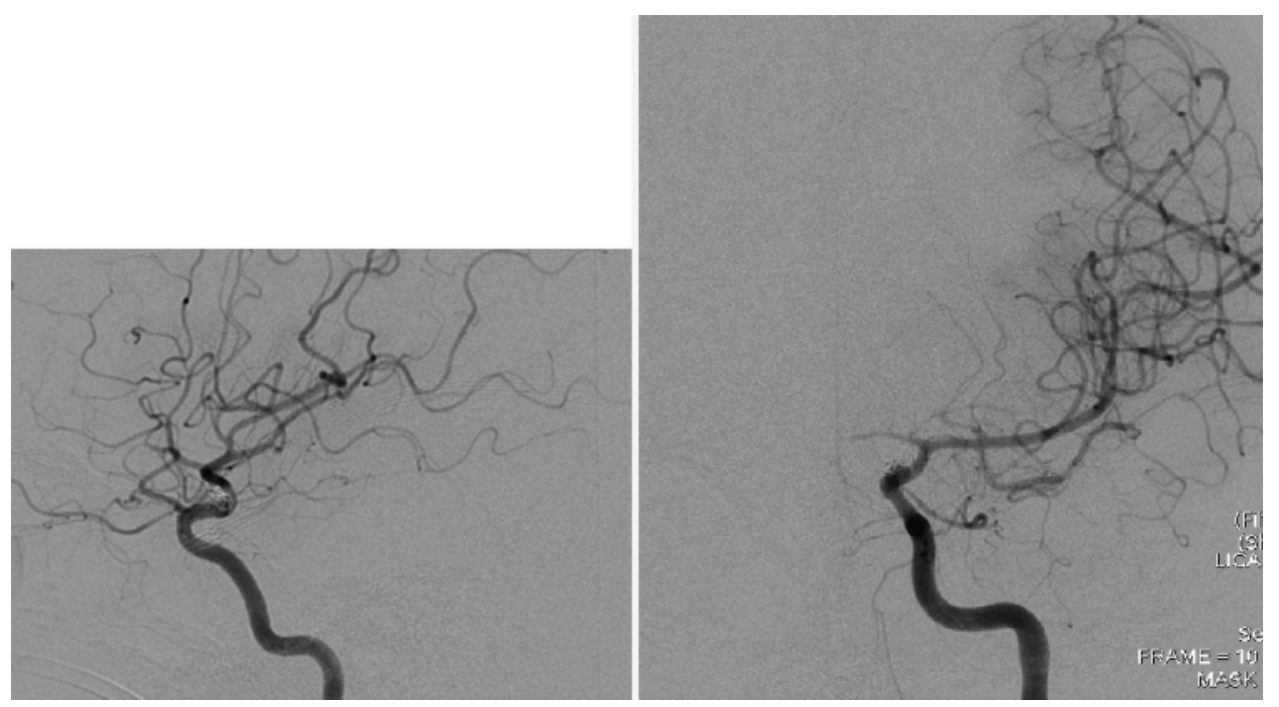

FIG. 3. Case 1. Lateral (left) and anteroposterior (right) DS angiograms demonstrating post-endovascular balloon remodeled coil embolization of the BBA. 

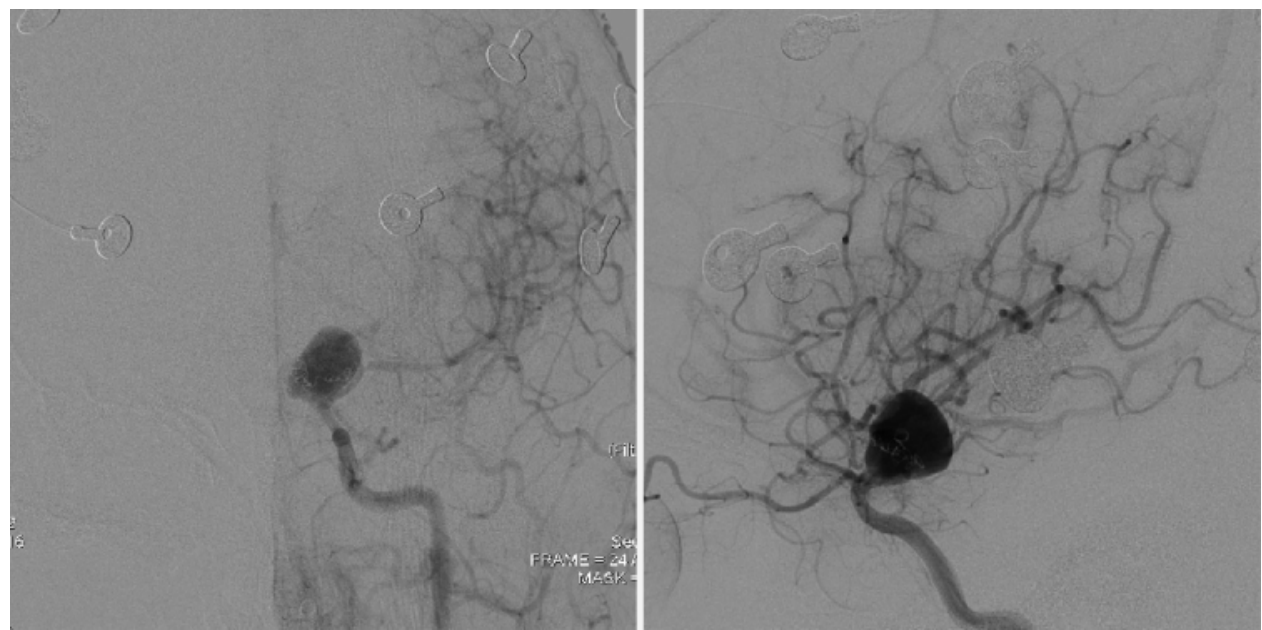

FIG. 4. Case 1. Anteroposterior (left) and lateral (right) DS angiograms demonstrating recurrent enlarging BBA after coiling.

appeared to be occluding the AChA origin, there was flow present on micro-Doppler ultrasonography. Although the PCoA was clipped at its origin, the PCoA perforating vessels were preserved and were supplied via the PCA.

Postoperative Course. A postoperative angiogram confirmed patent bypass and trapping of the aneurysm (Fig. 5). He eventually made an excellent recovery and has returned to work with minimal word-finding difficulties and very mild hemiparesis.

\section{Case 2}

Presentation and Examination. This 57-year-old man initially presented to an outside hospital with severe headache for which he was given an injection of ketorolac and discharged from the hospital. Later that day, he became unresponsive (Hunt and Hess Grade IV), and a head CT demonstrated a Fisher Grade 3 SAH. Cerebral angiography demonstrated a 3-mm BBA of the anterior wall of the ICA (Fig. 6).

First Operation. The patient underwent endovascular treatment consisting of dual stent remodeled coiling (Fig. 7). These stents were placed such that they overlapped completely not only to remodel the neck of the aneurysm, but also to function as a flow diverter. The patient experienced rehemorrhaging later that day; the distribution of blood was the same as that of his prior hemorrhage.
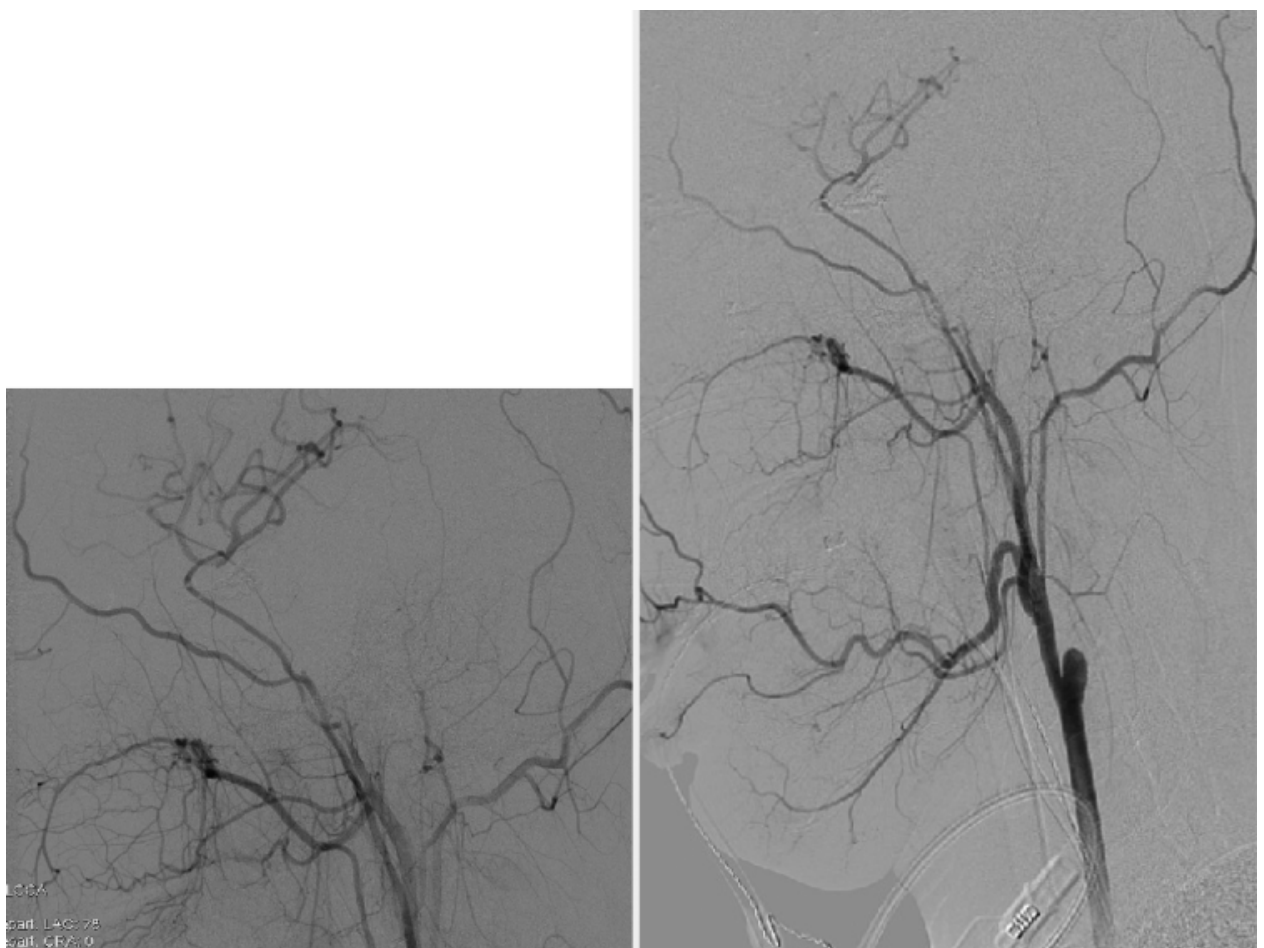

FIG. 5. Case 1. Lateral (left) and anteroposterior (right) DS angiograms obtained after EC- IC bypass and trapping. 


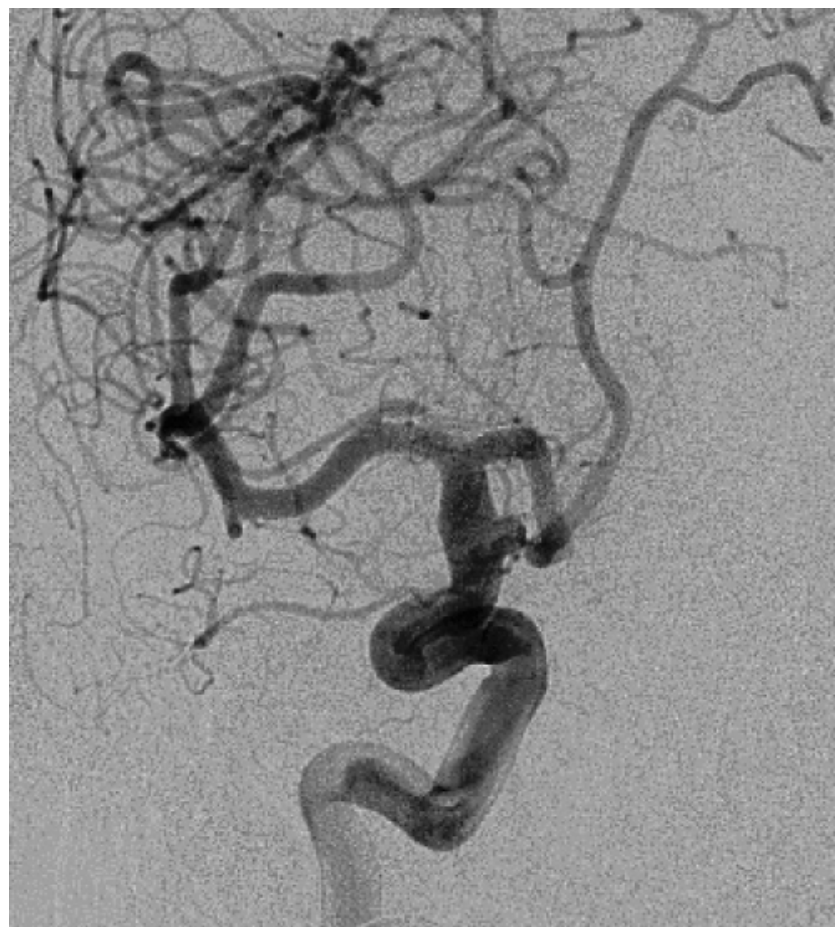

FIG. 6. Case 2. Digital subtraction angiogram demonstrating a 3-mm BBA of the anterior wall of the ICA.

Second Operation. The patient underwent decompressive craniectomy and evacuation of right frontal intraparenchymal hemorrhage. The antiplatelet effects of aspirin and clopidogrel were reversed with a platelet transfusion prior to and during the operation.

Postoperative Course. Postoperatively, the patient was maintained on $325 \mathrm{mg}$ aspirin daily, and the clopidogrel was discontinued. In the subsequent days, the patient underwent 5 interventional treatments for severe vasospasm including verapamil and balloon angioplasty. During endovascular treatment for vasospasm, there was no opacification of the aneurysm. On posthemorrhage Day 21, the patient experienced rehemorrhaging a second time and underwent an ECA- $\mathrm{M}_{2}$ high-flow bypass.

Brain edema precluded surgical trapping at the time of his craniotomy, and the patient immediately underwent postoperative endovascular parent vessel occlusion to include the aneurysm neck. This was carried out from the carotid artery terminus distally and proximally extended to the anterior genu of the ICA. In this case, the patient had already suffered multiple infarcts and brain injury secondary to vasospasm and the initial hemorrhage; therefore, preservation of the AChA was not crucial. The patient's condition failed to improve, despite further aggressive treatment of vasospasm with angioplasty and intraarterial verapamil infusion. Care was withdrawn 2 weeks later and the patient died.

\section{Case 3}

Presentation and Examination. This 24-year-old man developed sudden loss of consciousness. A CT scan demonstrated diffuse SAH and hydrocephalus. On admission, he

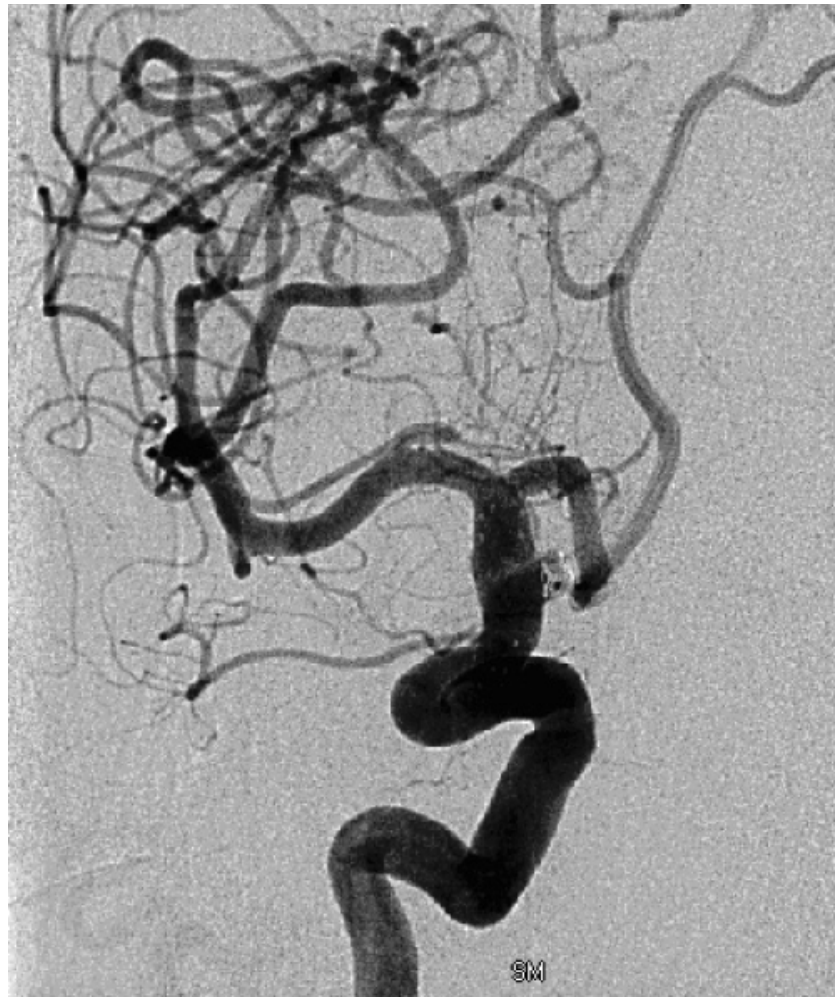

FIG. 7. Case 2. Digital subtraction angiogram obtained after coil placement with stent remodeling of the $3-\mathrm{mm} \mathrm{BBA}$.

had a Hunt and Hess grade of V. His clinical grade improved to a Grade IV after ventriculostomy placement. Diagnostic angiography revealed a 1-2-mm BBA of the posterior wall of the communicating segment of the ICA (Fig. 8). Definitive surgical or endovascular treatment was considered, but the patient was not a candidate for endovascular treatment due to the small size of the aneurysm. Eventually, the patient developed vasospasm and hemiplegia on Day 4 after hemorrhage, with striatal MCA territory infarcts. Medical and endovascular methods were used to treat cerebral vasospasm. After 2 weeks, the patient improved to the point that he was confused and had a mild hemiparesis.

Operation. On Day 21 posthemorrhage, he underwent an ECA- $\mathrm{M}_{2}$ bypass. Microsurgical trapping was precluded by cerebral edema; therefore, endovascular trapping was performed (Fig. 9). Endovascular coil occlusion of the ICA was performed distal to the origin of the ophthalmic artery and proximal to the origin of the AChA. The likely hypoplastic PCoA was never visible on angiography and, therefore, coil placement was performed across the origin of the PCoA.

Postoperative Course. The patient continued to improve after surgery and participated in inpatient rehabilitation. At the 6-week follow-up, the patient had no motor deficits and was considering a return to college (mRS Score 1).

\section{Case 4}

Presentation and Examination. This 56-year-old woman was unconscious at presentation (Hunt and Hess Grade IV) after a high-speed motor vehicle accident. The initial head 


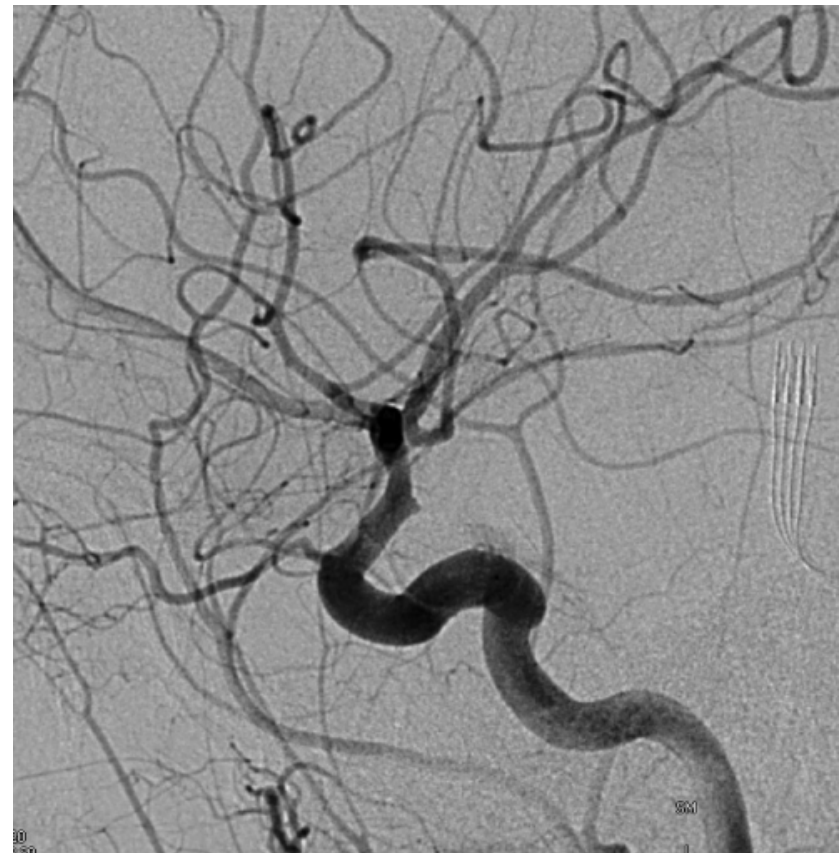

FIG. 8. Case 3. Digital subtraction angiogram demonstrating a BBA of the posterior wall of the communicating segment of the ICA.

CT demonstrated diffuse SAH. An angiogram revealed a submillimetric BBA of the ophthalmic segment of the ICA (Fig. 10). Ventriculostomy was performed, and the patient's condition improved 3-4 days later. She was following commands with symmetric strength, but continued to be lethargic and confused. Cerebral vasospasm was treated medically and endovascularly with intraarterial verapamil.

Operation and Postoperative Course. She underwent an ECA- $\mathrm{M}_{2}$ bypass performed on Day 15 after hemorrhage (Fig. 11). Microsurgical trapping was performed proximally with cervical ICA ligation, given the proximity of the aneurysm to the skull base. The distal clip was placed immediately proximal to the PCoA origin. Postoperative angiography demonstrated a robust construct without flow to the dysplastic aneurysm segment of the ICA. The patient's condition improved after inpatient rehabilitation and at the 1-month follow-up she had made an excellent recovery (mRS Score 1).

\section{Results}

All patients presented with poor clinical grade SAH and Hunt and Hess Grade IV. The Fisher grade based on imaging findings was severe (Grade 3 ) in all patients with the highest risk of vasospasm. Three of the patients were treated with a neuroendovascular procedure and various combinations of methods including primary coil occlusion, balloon-assisted coil embolization, and placement of multiple stents to divert flow away from the aneurysm. Symptomatic vasospasm developed in all patients, and after hypertensive hypervolemic hemodilution therapy (that is, triple$\mathrm{H}$ therapy) failed, they underwent endovascular treatment with intraarterial verapamil infusion and percutaneous balloon angioplasty. The vasospasm seemed to be particularly recalcitrant in these patients, and they underwent between

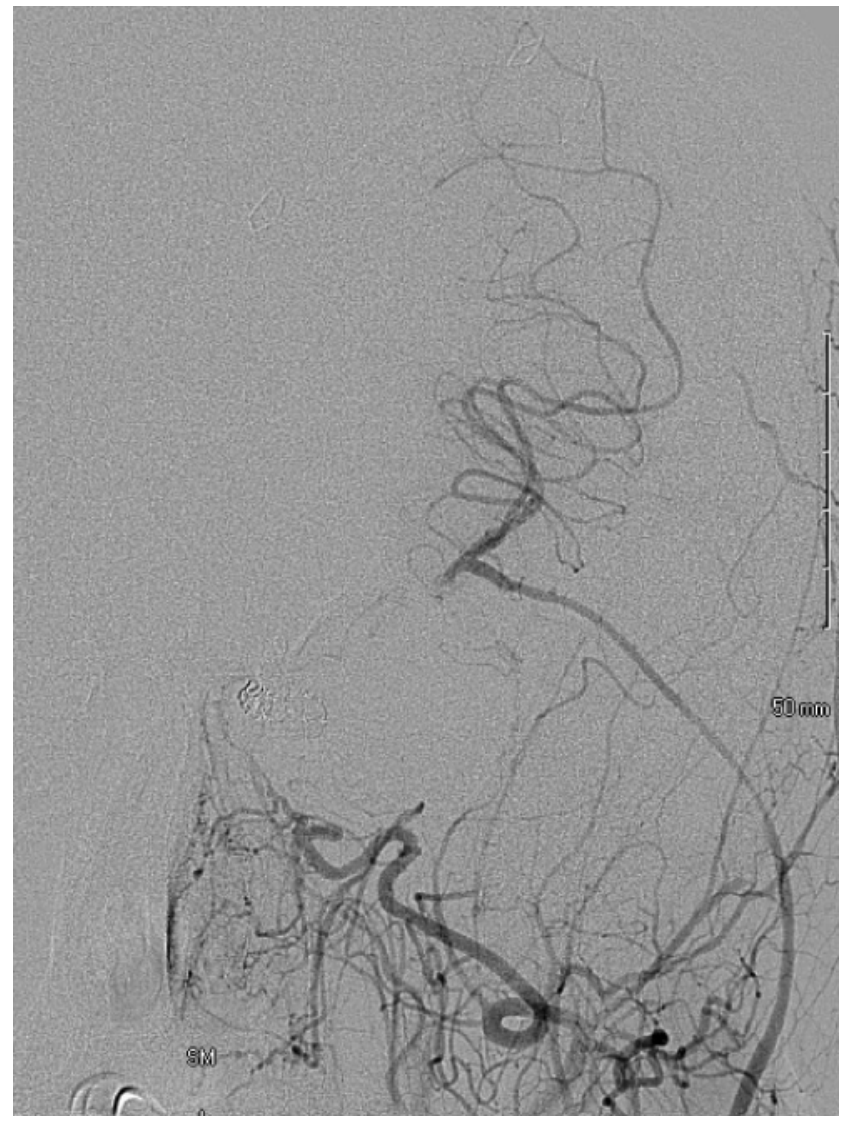

FIG. 9. Case 3. Anteroposterior DS angiogram demonstrating a patent graft after trapping and ECA-M $\mathrm{M}_{2}$ bypass.

2 and 9 separate procedures. An EC-IC bypass was performed, and complete aneurysm occlusion was demon-

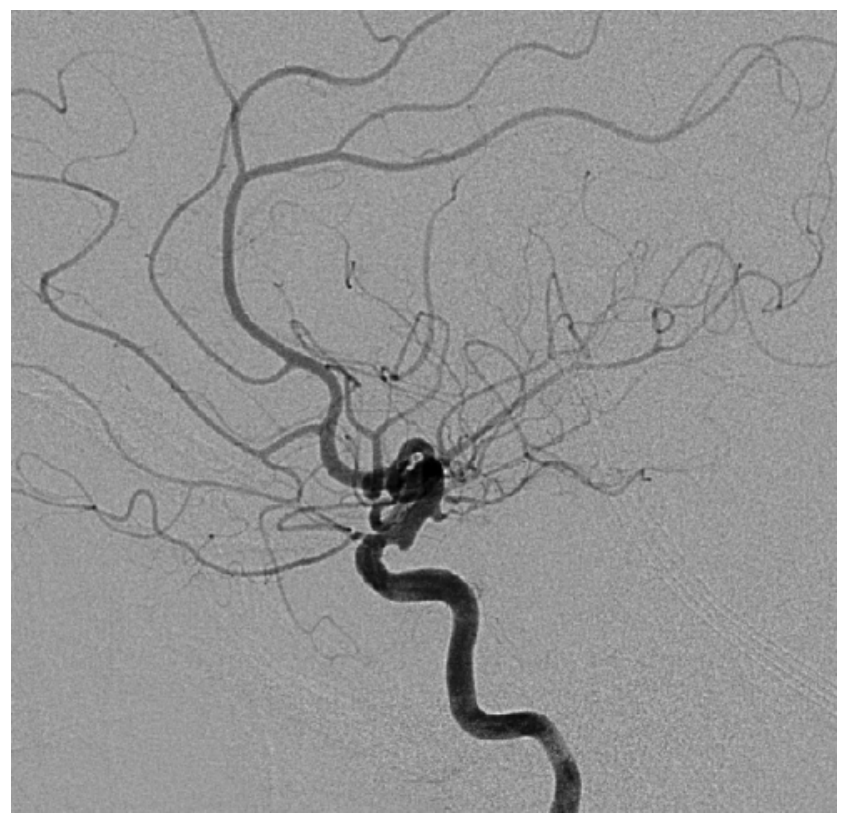

FIG. 10. Case 4. Lateral DS angiogram demonstrating a BBA of the ophthalmic segement of the ICA. 


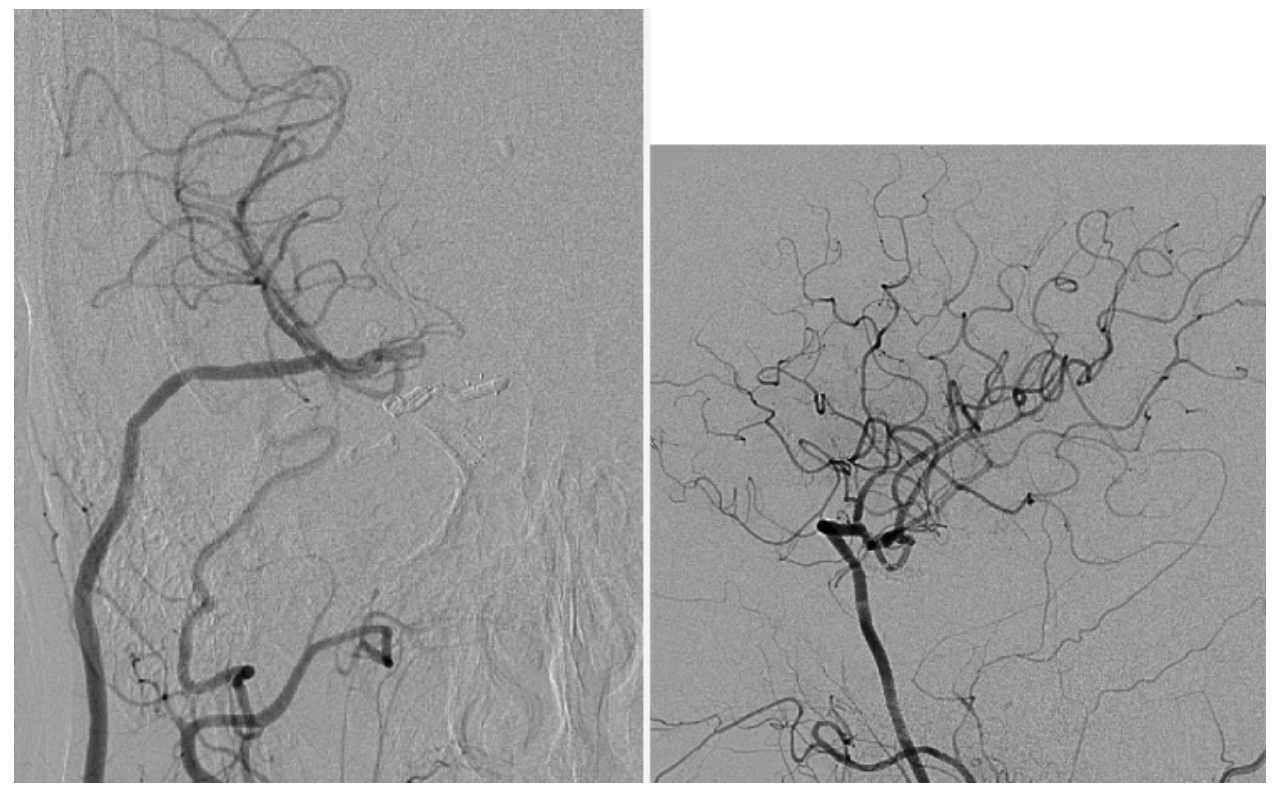

FIG. 11. Case 4. Anteroposterior (left) and lateral (right) DS angiograms demonstrating ECA- $\mathrm{M}_{2}$ bypass and microsurgical trapping of aneurysm.

strated on DS angiograms following bypass and trapping in all cases. The clinical outcome was good in 3 patients (mRS Score 1). The other patient experienced rehemorrhaging twice despite having undergone initial stent and coil placement. Care was withdrawn in this patient after his condition failed to improve after his third episode of SAH.

\section{Discussion}

Blood blister-like aneurysms originate in the supraclinoid ICA; however, they do not arise at branching points. These aneurysms may be confused with ophthalmic or superior hypophysial aneurysms. ${ }^{9}$ It is important to recognize these aneurysms because their treatment is certainly more difficult than that for saccular aneurysms of the supraclinoid ICA. The difficulty of treatment has been documented in the literature with case series and reports of "novel" surgical treatment methods. . $-7,17,19^{-19}$

Blood blister-like aneurysms of the ICA pose a unique challenge to treatment in cerebrovascular neurosurgery. Their small size, ill-defined necks, and thin fragile walls make these aneurysms particularly difficult to treat. ${ }^{4} \mathrm{Mul}-$ tiple strategies for primary treatment have been attempted including surgical clip placement, wrapping, clip placement after wrapping, endovascular coil embolization with or without adjunctive devices such as stents, or any combination of these. ${ }^{3,9-12,16,20}$ Primary treatment of BBAs surgically and endovascularly is associated with high morbidity and mortality rates and usually does not provide lasting results. In contrast, saccular aneurysms at nonbranching sites may be amenable to clip or coil embolization. The BBA's fragile walls are tenuous as evidenced by an intraoperative rupture that occurred after the bypass procedure had been completed in 1 of our cases (Case 1). Interestingly, similar to the case reported by Yanagisawa et al. ${ }^{19}$ proximal control was obtained by temporary clip application to the cervical ICA, and distal control was then ob- tained quickly by placing temporary clips. In our case, however, the aneurysm wall appeared to consist solely of fibrin clot with a coil mass traversing the vessel wall. Also, rapid enlargement of these aneurysms has been documented. ${ }^{3,11,12}$ Two of the 4 patients in the current series demonstrated enlargement of their aneurysms. One of these patients in particular demonstrated a marked enlargement of the aneurysm, which initially measured $3 \mathrm{~mm}$ and 2 weeks later was found to be $1 \mathrm{~cm}$.

Although anecdotal cases exist of successful, durable results, numerous authors have demonstrated a preponderance of failures when attempting to treat these aneurysms primarily with surgical clip application or endovascular coil embolization..$^{6,712,19,20}$ In light of these data and our recent experience, the most definitive method of treating these high-risk aneurysms is EC-IC bypass with parent vessel sacrifice. This is the only treatment that has consistently demonstrated durable results. Either proximal and distal clip placement or endovascular coil occlusion across the aneurysm segment has been shown to be efficacious in preventing rebleeding. One patient who underwent coil occlusion rebled twice despite coil and stent placement, and eventually care was withdrawn.

Typically, the decision for bypass is dependent on the results of BTO; however, in a patient with high-grade SAH, this is often not feasible. ${ }^{2}$ Although BTO was considered in our cases, the patients' neurological status, depressed level of consciousness, and neurological deficits (for example, hemiparesis) precluded meaningful analysis. Angiographic assessments were made, however, via balloon occlusion, regarding the extent of the collateral circulation. Patients with good clinical grade SAH (Hunt and Hess Grades I and II) can be better evaluated clinically with BTO. With this clinical information, aggressive treatment with bypass may be avoided if the patient does not have a change in neurological status during BTO. Single photon emission computed tomography may help to further characterize which 
patients require EC-IC bypass procedures; however, we did not use this technique in the patients in this series..$^{17,18}$

The methods of aneurysm trapping after EC-IC bypass include microsurgical clip placement or endovascular coil occlusion. The decision regarding which procedure to choose is multifactorial and must be tailored to the clinical and surgical scenario. Both methods have benefits, drawbacks, and a proper place in therapy. The location of the aneurysm, extent of cerebral edema, presence of a fetal circulation, clinical status, and neurological examination all factor into the decision. The method of trapping did not seem to affect the clinical outcome. Surgical trapping was accomplished with microsurgical clips in 2 patients. In the other 2 patients, trapping was accomplished endovascularly because further surgical dissection and retraction of edematous brain was thought to be detrimental. If there is a fetal PCoA, other bypass options must be considered. If the PCoA does not make a significant contribution to the PCA and the aneurysm is not involving the origin, the PCoA can be clipped at the origin with continued perfusion of the important PCoA perforatoring vessels via the $\mathrm{P}_{1}$ segment of the PCA (Case 1). Last, if precise trapping is necessary, microsurgical trapping is favored because endovascular coil placement involves a longer segment of the parent vessel compared with the relatively discreet placement of a clip.

\section{Conclusions}

Treatment of BBAs of the ICA remains difficult, particularly in the setting of high-grade SAH. These challenging patients often require multiple treatments and have a high incidence of rebleeding. In our experience, definitive treatment of these aneurysms consists of EC-IC bypass and surgical or endovascular trapping. Longer follow-up and a larger patient population will help elucidate the optimal treatment for patients with SAH due to BBAs.

\section{References}

1. Abe M, Tabuchi K, Yokoyama H, Uchino A: Blood blisterlike aneurysms of the internal carotid artery. J Neurosurg 89: 419-424, 1998

2. Abud DG, Spelle L, Piotin M, Mounayer C, Vanzin JR, Moret J: Venous phase timing during balloon test occlusion as a criterion for permanent internal carotid artery sacrifice. AJNR Am J Neuroradiol 26:2602-2609, 2005

3. Charbel FT, Gonzales-Portillo G, Hoffman W, Cochran E: Distal internal carotid artery pseudoaneurysms: technique and pitfalls of surgical management: two technical case reports. Neurosurgery 45:643-649, 1999

4. Ishikawa T, Nakamura N, Houkin K, Nomura M: Pathological conideration of a "blister-like" aneurysm at the superior wall of the internal carotid artery: case report. Neurosurgery 40: 403-406, 1997

5. Islam MS, Manabe H, Hasegawa S, Takemura A, Nagahata M: Successful staged treatment for ruptured blister-like dissecting aneurysm of the intracranial internal carotid artery: acute GDC embolization for the blister-like aneurysm followed by proximal occlusion with extracranial-intracranial bypass in the chronic stage. Minim Invasive Neurosurg 47:165-168, 2004

6. Joo SP, Kim TS, Moon KS, Kwak HJ, Lee JK, Kim JH, et al: Arterial suturing followed by clip reinforcement with circumferential wrapping for blister-like aneurysms of the internal carotid artery. Surg Neurol 66:424-429, 2006

7. Kubo Y, Ogasawara K, Tomitsuka N, Otawara Y, Watanabe M, Ogawa A: Wrap-clipping with polytetrafluoroethylene for ruptured blisterlike aneurysms of the internal carotid artery. Technical note. J Neurosurg 105:785-787, 2006

8. McNeely PD, Clarke DB, Baxter B, Vandorpe RA, Mendez I: Endovascular treatment of a "blister-like" aneurysm on the internal carotid artery. Can J Neurol Sci 27:247-250, 2000

9. Nakagawa F, Kobayashi S, Takemae T, Sugita K: Aneurysms protruding from the dorsal wall of the internal carotid artery. J Neurosurg 65:303-308, 1986

10. Ogawa A, Suzuki M, Ogasawara K: Aneurysms at nonbranching sites in the surpaclinoid portion of the internal carotid artery: internal carotid artery trunk aneurysms. Neurosurgery 47:578-586, 2000

11. Park JH, Park IS, Han DH, Kim SH, Oh CW, Kim JE, et al: Endovascular treatment of blood blister-like aneurysms of the internal carotid artery. J Neurosurg 106:812-819, 2007

12. Pelz DM, Ferguson GG, Lownie SP, Kachur E: Combined endovascular/neurosurgical therapy of blister-like distal internal carotid aneurysms. Can J Neurol Sci 30:49-53, 2003

13. Rankin J: Cerebral vascular accidents in patients over the age of 60. II. Prognosis. Scott Med J 2:200-215, 1957

14. Redekop GJ, Woodhurst B: Unusual aneurysms of the distal internal carotid artery. Can J Neurol Sci 25:202-208, 1998

15. Segal DH, Sen C, Bederson JB, Catalano P, Sacher M, Stollman AL, et al: Predictive value of balloon test occlusion of the internal carotid artery. Skull Base Surg 5:97-107, 1995

16. Shigeta H, Kyoshima K, Nakagawa F, Kobayashi S: Dorsal internal carotid artery aneurysms with special reference to angiographic presentation and surgical management. Acta Neurochir (Wien) 119:42-48, 1992

17. Sim SY, Shin YS, Cho KG, Kim SY, Kim SH, Ahn YH, et al: Blood blister-like aneurysms at nonbranching sites of the internal carotid artery. J Neurosurg 105:400-405, 2006

18. Yamamoto Y, Nishiyama Y, Toyama Y, Satoh K, Irie K, Ohkawa M: Preliminary results of Tc-99m ECD SPECT to evaluate cerebral collateral circulation during balloon test occlusion. Clin Nucl Med 27:633-637, 2002

19. Yanagisawa T, Mizoi K, Sugawara T, Suzuki A, Ohta T, Higashiyama N, et al: Direct repair of a blisterlike aneurysm on the internal carotid artery with vascular closure staple clips. Technical note. J Neurosurg 100:146-149, 2004

20. Yanaka K, Meguro K, Nose T: Repair of a tear at the base of a blister-like aneurysm with suturing and an encircling clip: technical note. Neurosurgery 50:218-221, 2002

Manuscript submitted November 15, 2007.

Accepted December 10, 2007.

Address correspondence to: Mustafa K. Başkaya, M.D., Department of Neurological Surgery, Clinical Science CenterK4/828, 600 Highland Avenue, Madison, Wisconsin 53792. email: m.baskaya@neurosurg.wisc.edu. 